\title{
Capacity of Inhomogeneous Hybrid Wireless Networks in Three-Dimensional Space
}

\author{
http://dx.doi.org/10.3991/ijoe.v11i9.5064 \\ Feng Wu, Jiang Zhu, Yilong. Tian and Zhipeng Xi \\ National University of Defense Technology, Changsha, China
}

\begin{abstract}
Network capacity has been widely studied in recent years. However, most of the literatures focus on the networks where nodes are distributed in a two-dimensional space. In this paper, we propose a 3D hybrid sensor network model. By setting different sensor node distribution probabilities for cells, we divide all the cells in the network into dense cells and sparse cells. Analytical expressions of the aggregate throughput capacity are obtained. We also find that suitable inhomogeneity can increase the network throughput capacity.
\end{abstract}

Index Terms-Network Capacity; Hybrid; Inhomogeneity; Wireless Sensor Networks

\section{INTRODUCTION}

Network capacity is one of the most important issues in wireless sensor networks. Gupta and Kumar initiate the study of network capacity in [1], they find that in a wireless network where all the nodes are randomly distributed, the per-node throughput capacity is $\Theta\left(\frac{W}{\sqrt{n \log n}}\right)$, where $W$ is the total bandwidth and $n$ is the number of nodes. They prove that the per-node throughput will decrease to zero as the number of nodes goes to infinity, which is a pessimistic conclusion.

Following this work, extensive studies have been conducted to achieve a tighter capacity bound [2-8]. Franceschetti et al. [2] apply percolation theory to obtain a per-node transmission rate higher than $\Theta(1 / \sqrt{n})$. Grossglauser and Tse [3] prove that network capacity can be increased with mobility of ad hoc nodes. They show that per-node transmission rate of $\Theta(W)$ can be achieved when ad hoc nodes are mobile, while the transmission delay will go to infinity.

Few researchers propose to add some base stations to increase network capacity, and they call this kind of networks 'hybrid networks' [9-11]. Liu et al. [9] study the capacity of hybrid networks where base stations are regularly placed. They prove that the per-node throughput capacity can be improved significantly with the help of base stations. Kozat et al. [10] consider the case that base stations are randomly placed and prove that the per-node throughput capacity is decided by the number of base stations. Zemlianov et al. [11] propose a network model where base stations are arbitrarily placed and they get a similar conclusion.
However, most of the literatures focus on the homogeneous networks, where all the nodes are uniformly distributed. In most real cases, networks exhibit inhomogeneity. A few works have analyzed the capacity of inhomogeneous networks [12-14]. Perevalov et al. [12] study the capacity of a clustered wireless networks. Alfano et al. $[13,14]$ consider nodes are placed to a shotnoise Cox process (SNCP). They obtain the upper bound and lower bound of their network model.

The aforementioned works on inhomogeneous networks are based on the assumption that all the nodes are distributed in a $2 \mathrm{D}$ space. With the development of technology, the wireless networks are extending from $2 \mathrm{D}$ space to 3D space. To the best of our knowledge, few studies focus on the 3D wireless networks [15-16]. In [15], Gupta and Kumar study the transport capacity of 3D arbitrary networks and the throughput capacity of 3D random networks. Pan et al. [16] consider the case that the node spatial distributions are inhomogeneous.

In this paper, we for the first time propose a 3D hybrid network model characterized by inhomogeneity and obtain the analytical expressions of network capacity. By setting different distribution probabilities for cells, we divide all the cells into dense cells and sparse cells. On this basis, we derive the capacity of dense cells and sparse cells respectively. Then we calculate the aggregate throughput capacity and analyze the results we obtain. We find that suitable inhomogeneity can increase the network capacity, which is an interesting conclusion.

The remainder of this paper is organized as follows. In Section II, we present a 3D inhomogeneous hybrid network model. In Section III, we derive the aggregate throughput capacity of our network model and analyze the capacity we get. Finally, we conclude our studies in Section IV.

\section{NETWORK MODEL}

\section{A. Network Architecture}

We consider a wireless sensor network with $n$ sensor nodes and $m$ base stations distributed in a threedimensional cube of unit volume. We assume that the base stations are regularly placed, hence the unit cube is divided into $\sqrt[3]{m} \times \sqrt[3]{m} \times \sqrt[3]{m}$ equal-sized cubelets, (we assume that $\sqrt[3]{m}$ is integer for simplicity of analysis). We label them $\{(i, j, k): i, j, k=1, \ldots, \sqrt[3]{m}\}$. Each base stations lies into the center of the cell where it is, as shown in fig.1. 
The base station is connected by a wired network, hence we can assume that the bandwidth is not constrained.

To make our network model exhibit inhomogeneity, we set different node distribution probabilities for each cell. Concretely, for cell $k(1 \leq k \leq m)$, the probability that each node are distributed in it is $p_{k}$, hence we have $\sum_{k=1}^{m} p_{k}=1$. Then, for the sensor nodes in the same cell, we assume that they are independent and identically (i.i.d.). Though the distribution of sensor nodes in a certain cell is homogeneous, the overall node spatial distribution exhibits inhomogeneity.

Specially, when $p_{1}=p_{2}=\ldots=p_{m}=\frac{1}{m}$, our network model is an homogeneous model. Thus, our results obtained is more usual than that of the homogeneous networks.

\section{B. Communication Model}

In this paper, we adopt the protocol model proposed in [1] as our communication model. Let $r$ denote the transmission range of sensor nodes, node $X_{j}$ can receive the packets from node $X_{i}$ successfully if the following conditions are satisfied:

(1) Node $X_{j}$ is under the coverage of node $X_{i}$, i.e.

$$
\left|X_{i}-X_{j}\right| \leq r
$$

(2) For an arbitrary other node $X_{t}$ which is simultaneously delivering over the same channel,

$$
\left|X_{t}-X_{j}\right| \geq(1+\Delta)\left|X_{i}-X_{j}\right|
$$

where $\Delta$ defines the size of guard zone.

\section{Routing Strategy}

In our network model, there are two types of transmission modes: multi-hop mode and infrastructure mode. In multi-hop mode, the sensor nodes communicate with each other in a multi-hop fashion. In infrastructure

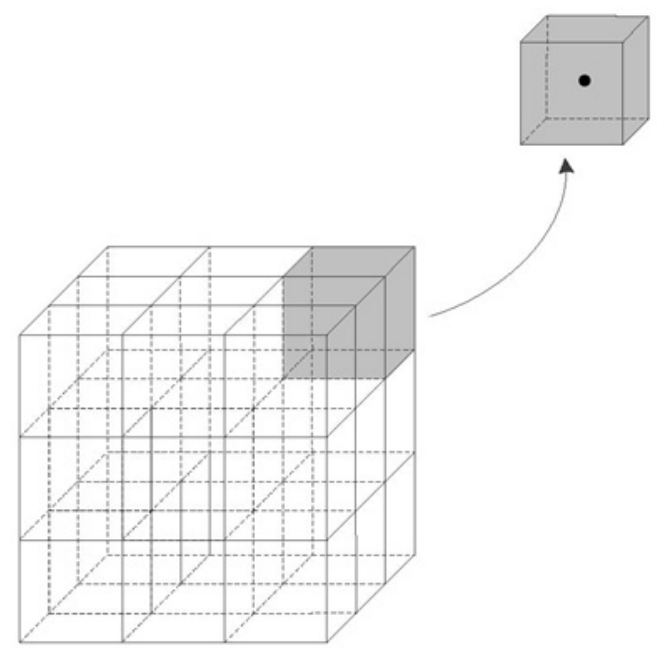

Figure 1. Network Architecture mode, the source node delivers packets to the destination node with the help of base stations.

In this paper, we use the 0 -nearest-cell routing strategy. Concretely, if the source node and the destination node are in the same cell, they communicate with each other in multi-hop mode. If the source node and the destination node are in different cells, they communicate in infrastructure mode.

We assume that the total bandwidth is $W$. According to the routing strategy, the total bandwidth is divided into three sub-channels: intra-cell sub-channels, uplink subchannels and downlink sub-channels. Let $W_{1}, W_{2}$ and $W_{3}$ denote the three types of sub-channels respectively, and we have $W_{1}+W_{2}+W_{3}=W$. As the amount of packets over uplink sub-channels are the same with that over downlink sub-channels, we let $W_{2}=W_{3}$.

\section{NETWORK CAPACITY}

\section{A. Dense Cells and Sparse Cells}

In the passage above we have introduced our network model. To make our network model exhibit inhomogeneity, we set distribution probabilities for different cells. According to the asymptotic behavior of distribution probabilities, we divide all the cells into two types: dense cells and sparse cells.

Definition 1: Dense cells. For cell $k$, if the node distribution probability $p_{k}=\Omega(1 / \sqrt{n})$, we say cell $k$ is a dense cell.

Definition 2: Sparse cells. For cell $l$, if the node distribution probability $p_{l}=o(1 / \sqrt{n})$, we say cell $l$ is a sparse cell.

Consider cell $k$, let $Y_{i}$ denote whether sensor node $i$ $(1 \leq i \leq n)$ and its destination node are both in cell $k$, i.e.

$Y_{i}=\left\{\begin{array}{rr}1, & \text { both in cell } k \\ 0, & \text { otherwise }\end{array}\right.$.

For each node, the probability that it is distributed in cell $k$ is $p_{k}$, hence we have $E\left[Y_{i}\right]=p_{k}^{2}$.

Let $N_{k}$ denote the number of source-destination pairs in cell $k$, then we have:

$$
\frac{N_{k}}{n}=\frac{1}{n} \sum_{i=1}^{n} Y_{i} \rightarrow p_{k}^{2} \text { as } n \rightarrow+\infty
$$

Thus, if $p_{k}=\Omega(1 / \sqrt{n})$, we have $\lim _{n \rightarrow \infty} p_{k}{ }^{2} n \rightarrow+\infty$, and if $p_{k}=o(1 / \sqrt{n})$, we have $p_{k}=o(1 / \sqrt{n})$.

The analysis above implies that in dense cells, the number of source-destination pairs $N_{k}$ is not constrained as $n$ goes to infinity, and in sparse cells, $N_{k}$ is constrained as $n$ goes to infinity.

\section{B. Capacity Contributed by Multi-Hop Mode}

In this section we derive the throughput capacity contributed by multi-hop mode. Since $W_{1}, W_{2}$ and $W_{3}$ are different sub-channels, there are no interference between them. However, interference exists between the same type 
of traffic in different cells. Reference [9] have proposed the notion of interfering neighbors and proved that in 2D hybrid networks, for an arbitrary cell, the number of its interfering neighbors is bounded by a constant. We adopt this notion to the $3 \mathrm{D}$ inhomogeneous hybrid networks and obtain a similar conclusion.

Definition 5: Interfering Neighbors. If there is a point in cell $l$ which is within a distance $(2+\Delta) r$ of some point in cell $k$, we say the two cells are interfering neighbors.

Theorem 1: In an inhomogeneous hybrid network in a 3D space, for each cell, the number of its interfering neighbors is bounded by $c, c$ is a constant only depends on $\Delta$.

Proof: We denote the length of each side of a cell (cubelet) as $a$ and assume that $a=b r$, where $b$ is a constant. Hence each cell is contained by a ball with radius $\frac{\sqrt{3}}{2} a$ and contains a ball with radius $\frac{1}{2} a$.

If a cell $l$ is an interfering neighbor of cell $k$, one point in cell $l$ must be within a distance $(2+\Delta) r$ of some point in cell $k$. Therefore, all the interfering neighbors of cell $k$ must be contained by a ball with radius $\frac{3 \sqrt{3}}{2} a+(2+\Delta) r$. Hence, the number of interfering neighbors is loosely bounded by

$$
\begin{aligned}
c & =\frac{4}{3} \pi\left[\frac{3 \sqrt{3}}{2} a+(2+\Delta) r\right]^{3} / a^{3} \\
& =\frac{4}{3} \pi\left(\frac{\frac{3 \sqrt{3}}{2} b+2+\Delta}{b}\right)^{3}
\end{aligned}
$$

By the theorem above, we can conclude that there is a spatial scheduling policy that each cell gets one slot to transmit data in every $(1+c)$ slots.

Theorem 2: If cell $k$ is a dense cell, its aggregate capacity contributed by multi-hop mode is

$$
T_{m, k}=\Theta\left(p_{k} \sqrt[3]{\frac{p_{k} n^{2}}{\log ^{2}\left(p_{k}^{2} n\right)}} W_{1}\right)
$$

where $p_{k}$ is the distribution probability of cell $k$.

Proof: Since cell $k$ is a dense cell, $p_{k}=\Omega(1 / \sqrt{n})$, the number of source-destination pairs in cell $k$ goes to infinity as $n$ goes to infinity. According to the conclusion of reference [15], with large enough $N_{k}$, the aggregate capacity contributed by multi-hop mode is:

$$
T=\Theta\left(\sqrt[3]{\frac{N_{k}^{2}}{\log ^{2} N_{k}}} W_{1}\right) .
$$

By (1), we know that $N_{k} \rightarrow p_{k}{ }^{2} n$ as $n$ goes to infinity. Hence, the aggregate capacity of cell $k$ contributed by multi-hop is:

$$
\begin{aligned}
T_{m, k} & =\Theta\left(\sqrt[3]{\frac{N_{k}^{2}}{\log ^{2} N_{k}}} W_{1}\right) \\
& =\Theta\left(\sqrt[3]{\left.\frac{\left(p_{k}^{2} n\right)^{2}}{\log ^{2}\left(p_{k}^{2} n\right)} W_{1}\right)} .\right. \\
& =\Theta\left(p_{k} \sqrt[3]{\frac{p_{k} n^{2}}{\log ^{2}\left(p_{k}{ }^{2} n\right)}} W_{1}\right)
\end{aligned}
$$

Theorem 3: If cell $l$ is a sparse cell, its aggregate capacity contributed by multi-hop mode is

$$
T_{m, l}=O\left(p_{l} \sqrt[3]{p_{l} n^{2}}\right)
$$

where $p_{l}$ is the distribution probability of cell $l$.

Proof: Since cell $l$ is a sparse cell, $p_{l}=o(1 / \sqrt{n})$, the number of source-destination pairs $N_{l}$ in cell $l$ is constrained as $n$ goes to infinity. We can not use the method in Theorem 2 to calculate the network capacity.

Reference [15] gives the transport capacity when a 3D network is optimally operated, the transport capacity is:

$$
\lambda n \bar{L} \leq 2\left(\frac{3 V}{4 \pi}\right) \frac{1}{\Delta} W n^{\frac{2}{3}} \text { bit-mete/sec }
$$

where $\lambda$ is the average per-node transmission rate, $\bar{L}$ is the average distance of packets transmission, $V$ is the volume of the space.

Consider a sparse cell $l$, the volume $V_{l}=\frac{1}{m}$, the and $\bar{L}=\Theta\left(\frac{1}{\sqrt[3]{m}}\right)$. Since (4) gives the transport capacity bound of an optimally operated network, which is larger than that of a random network, we have:

$$
T_{m}\left(N_{l}\right) \leq \lambda N_{k} \leq 2\left(\frac{3}{4 \pi}\right)^{\frac{1}{3}} \frac{1}{\Delta} W_{1} N_{l}^{\frac{2}{3}}=O\left(\sqrt[3]{N_{l}^{2}} W_{1}\right)
$$

Since $N_{l} \rightarrow p_{l}^{2} n$ as $n$ goes to infinity, we have:

$$
T_{m, l}=O\left(\sqrt[3]{\left(p_{l}^{2} n\right)^{2}} W_{1}\right)=O\left(p_{l} \sqrt[3]{p_{l} n^{2}}\right) .
$$

\section{Capacity Contributed by Infrastructure Mode}

Since the base infrastructures communicate through wired channels, their bandwidth assumed not to be constrained. The bandwidths of uplink and downlink subchannels are both $W_{2}$. Thus, the throughput capacity is bounded by $W_{2}$. By the analysis above, we have the following theorem.

Theorem 4: In 3D inhomogeneous hybrid networks, the aggregate capacity contributed by infrastructure mode is:

$$
T_{i}=\Theta\left(m W_{2}\right) .
$$

\section{Aggregate Capacity of the Whole Networks}

Now we calculate the aggregate throughput capacity of the whole networks. The whole networks consist of dense 
cells and sparse cells. For simplicity, we let $m_{d}$ denote the number of dense cells, $m_{s}$ denote the number of sparse cells. Thus we have $m_{d}+m_{s}=m$. The following theorem shows the relationship of $m_{d}$ and $m$.

Theorem 5: If $m=o(\sqrt{n})$, we have $m_{d} \geq 1$; if $m=\Omega(\sqrt{n})$, we have $m_{d}=o(m)$.

Proof: For simplicity, we assume that cells $1,2, \ldots, l$ are dense cells, cells $l+1, \ldots m$ are sparse cells. Therefore, we have:

$$
\begin{aligned}
& \lim _{n \rightarrow \infty} p_{i} \sqrt{n}=\left\{\begin{array}{l}
\infty \quad(i=1, \ldots l) \\
\text { const. }(i=l+1, \ldots m)
\end{array}\right. \\
& \text { Let } \overline{n_{d}}=\frac{\sum_{i=1}^{l} p_{i} \sqrt{n}}{m_{d}}, \overline{n_{s}}=\frac{\sum_{i=l+1}^{m} p_{i} \sqrt{n}}{m_{s}}, \quad \text { th } \\
& \text { If } m=o(\sqrt{n}), \text { we have: } \\
& \lim _{n \rightarrow \infty} \frac{\sqrt{n}}{m}=\lim _{n \rightarrow \infty} \frac{\left(p_{1}+\ldots+p_{m}\right) \sqrt{n}}{m}=\infty .
\end{aligned}
$$

It can be also written as:

$$
\lim _{n \rightarrow \infty} \frac{m_{d} \overline{n_{d}}+m_{s} \overline{n_{s}}}{m}=\infty .
$$

Since $\frac{m_{s}}{m} \leq 1$ and $\overline{n_{s}}=o(\sqrt{n})$, we have $m_{d} \neq 0$.

If $m=\Omega(\sqrt{n})$, similarly, $\lim _{n \rightarrow \infty} \frac{m_{d} \overline{n_{d}}+m_{s} \overline{n_{s}}}{m}$ is bounded by a constant. Thus we have $m_{d}=o(m)$.

The theorem above implies if the number of base stations is small, there is at least one dense cell. And if the number of base stations is large enough, the number of dense cells is limited.

Based on the analysis above, we have the following theorem.

Theorem 6: In 3D hybrid inhomogeneous network, if dense cell exists in the network $\left(m_{d} \neq 0\right)$, the aggregate capacity

$$
T_{m}=\Theta\left(\sum_{k=1}^{m_{d}} p_{k} \sqrt[3]{\frac{p_{k} n^{2}}{\log ^{2}\left(p_{k}{ }^{2} n\right)}} W_{1}\right)+O\left(\sum_{l=m_{d}+1}^{m} p_{l} \sqrt[3]{p_{l} n^{2}} W_{1}\right) \text { (6) }
$$

And if no dense cell exists in the network, the aggregate capacity is

$$
T=O\left(\sum_{l=1}^{m} p_{l} \sqrt[3]{p_{l} n^{2}} W_{1}\right)+\Theta\left(m W_{2}\right)
$$

Proof: If $m_{d} \neq 0$, by the conclusion of theorem 2 and theorem 3 , the aggregate throughput capacity contributed by multi-hop mode is

$$
\begin{aligned}
T_{m}= & \sum_{k=1}^{m_{d}} T_{m, k}+\sum_{l=m_{d}+1}^{m} T_{m, l} \\
= & \Theta\left(\sum_{k=1}^{m_{d}} p_{k} \sqrt[3]{\frac{p_{k} n^{2}}{\log ^{2}\left(p_{k}{ }^{2} n\right)}} W_{1}\right) \\
& +O\left(\sum_{l=m_{d}+1}^{m} p_{l} \sqrt[3]{p_{l} n^{2}}\right)
\end{aligned}
$$

Combining (5) and (8), we can obtain the aggregate capacity of the whole network:

$$
\begin{aligned}
T= & T_{m}+T_{i} \\
= & \Theta\left(\sum_{k=1}^{m_{d}} p_{k} \sqrt[3]{\frac{p_{k} n^{2}}{\log ^{2}\left(p_{k}^{2} n\right)}} W_{1}\right) \\
& +O\left(\sum_{l=m_{d}+1}^{m} p_{l} \sqrt[3]{p_{l} n^{2}} W_{1}\right)+\Theta\left(m W_{2}\right)
\end{aligned}
$$

If $m_{d}=0, p_{1}=p_{2}=\ldots=p_{k}=0$. Then the aggregate capacity contributed by multi-hop mode is:

$$
T_{m}=O\left(\sum_{l=1}^{m} p_{l} \sqrt[3]{p_{l} n^{2}}\right)
$$

The aggregate capacity of the whole network is:

$$
T=O\left(\sum_{l=1}^{m} p_{l} \sqrt[3]{p_{l} n^{2}} W_{1}\right)+\Theta\left(m W_{2}\right)
$$

\section{E. Capacity Analysis}

In this section we discuss how the inhomogeneity affects the network capacity. Concretely, in our network model, for different cells, the distributions probabilities are different. Now we assume that all the dense cells have the same distribution probability, and so do the sparse cells. Then we have the following corollaries.

Corollary 1: In 3D hybrid network, if all the dense cells have the same distribution probability ( $\left.p_{1}=p_{2}=\ldots=p_{m_{d}}=\overline{p_{d}}\right)$, and so do the sparse cells $\left(p_{m_{d}+1}=p_{m_{d}+2}=\ldots p_{m}=\overline{p_{s}}\right)$, the aggregate throughput capacity of the whole networks is:

$$
T_{m}^{\prime}=\Theta\left(m_{d} \overline{p_{d}} \sqrt[3]{\frac{\overline{p_{d}} n^{2}}{\log ^{2}\left({\overline{p_{d}}}^{2} n\right)}}\right)+O\left(m_{s} \overline{p_{s}} \sqrt[3]{\overline{p_{s}} n^{2}}\right)
$$

Corollary 2: The aggregate capacity of a network where $p_{1} \neq p_{2} \ldots \neq p_{m_{d}}$ and $p_{m_{d}+1} \neq p_{m_{d}+2} \neq \ldots \neq p_{m}$ is $T_{m}$. If there is another network where all the dense cells have the same distribution probability $\overline{p_{d}}=\frac{\sum_{k=1}^{m_{d}} p_{k}}{m_{d}}$ and so do the sparse cells $\overline{p_{s}}=\frac{\sum_{l=m_{d}+1}^{m} p_{l}}{m_{s}}$, its aggregate capacity is $T_{m}{ }^{\prime}$. We have:

$$
T_{m}>T_{m}^{\prime}
$$


Proof: Let $f(x)=x \sqrt{\frac{x n^{2}}{\log ^{2}\left(x^{2} n\right)}}$. According to $\mathrm{L}^{\prime}$

Hospital rule, as $n$ goes to infinity, we have:

$$
\begin{aligned}
\lim _{n \rightarrow \infty} f(x) & =\sqrt[3]{\lim _{n \rightarrow \infty} \frac{x^{4} n^{2}}{\log ^{2}\left(x^{2} n\right)}} \\
& =\sqrt[3]{\lim _{n \rightarrow \infty} \frac{\left(x^{4} n^{2}\right)_{n}^{\prime}}{\left(\log ^{2}\left(x^{2} n\right)\right)_{n}^{\prime}}} \\
& =\sqrt[3]{\lim _{n \rightarrow \infty} \frac{2 x^{4} n}{2 \log \left(x^{2} n\right) \cdot x^{2} \cdot \frac{1}{x^{2} n}}} \\
& =\sqrt[3]{\lim _{n \rightarrow \infty} \frac{x^{4} n^{2}}{\log \left(x^{2} n\right)}} \\
& =\sqrt[3]{\frac{\left(x^{4} n^{2}\right)_{n}^{\prime}}{\left(\log \left(x^{2} n\right)\right)_{n}^{\prime}}} \\
& =\sqrt[3]{x^{4} n^{2}}
\end{aligned}
$$

Therefore, when $n$ is large enough, $f(x)$ is a concave function. We have:

$$
f\left(\sum_{k=1}^{m_{d}} p_{k}\right)>m_{d} f\left(\frac{\sum_{k=1}^{m_{d}} p_{k}}{m_{d}}\right) .
$$

Hence we can conclude:

$$
\sum_{k=1}^{m_{d}} p_{k} \sqrt[3]{\frac{p_{k} n^{2}}{\log ^{2}\left(p_{k}{ }^{2} n\right)}} W_{1}>m_{d} \overline{p_{d}} \sqrt[3]{\frac{\overline{p_{d}} n^{2}}{\log ^{2}\left({\overline{p_{d}}}^{2} n\right)}} W_{1} .
$$

Similarly, let $g(x)=x \sqrt[3]{x n^{2}}$. Since $g(x)$ is also a concave function, we have:

$$
\sum_{l=m_{d}+1}^{m} p_{l} \sqrt[3]{p_{l} n^{2}} W_{1}>m_{s} p_{s} \sqrt[3]{p_{s} n^{2}} W_{1}
$$

Combining (6), (10), (11) and (12), we have:

$$
T_{m}>T_{m}^{\prime} \text {. }
$$

The corollary above implies that in 3D inhomogeneous hybrid network, if the same type of cells exhibit homogeneity, the network capacity will decrease. In other words, suitable inhomogeneity can increase the 3D network capacity.

\section{CONCLUSIONS}

In this paper, we propose an inhomogeneous hybrid network model where all the sensor nodes are distributed in a 3D space. By setting different sensor node distribution probabilities for cells, we divide all the cells into dense cells and sparse cells. We derive the aggregate throughput capacity of our network model and analyze the results we obtain. We find that under the same conditions, inhomogeneity can increase the network capacity.

\section{REFERENCES}

[1] G. Gupta, and P. R. Kumar, "The Capacity of Wireless Networks," IEEE Transactions on Information Theory, 2000, vol. 46, pp. 388-404. http://dx.doi.org/10.1109/18.825799

[2] M. Franceschetti, D. Douse, and D. Tse."Closing the Gap in the Capacity of Random Wireless Networks via Percolation Theory" IEEE Transactions on Information Theory, 2007, vol. 53, pp. 1009-1018. http://dx.doi.org/10.1109/TIT.2006.890791
[3] M. Grossglauser and D. Tse."Mobility Increases the Capacity of Ad Hoc Wireless Networks" IEEE Transactions on Networking, 2002, vol. 10, pp. 477-486. http://dx.doi.org/10.1109/TNET. 2002.801403

[4] S. Ning, J. Yoonsu and L. Sanho, “ Energy Efficient Mechanism using Flexible Medium Access Control Protocol for Hybrid Wireless Sensor Networks," Journal of Central South University, 2013, vol. 20, pp. 2165-2174. http://dx.doi.org/10.1007/s11771013-1721-6

[5] S. Toumpis, "Capacity Bounds for Three Types of Wireless Networks: Asymmetric, Cluster and Hybrid," Proc. ACM MobiHoc, Roppongi, Japan, 2004, pp. 133-144.

[6] C. Jiang, Y. Chen, Y. Ren and J. Ray. "Maximizing Network Capacity with Optimal Source Selection: A Network Science Perspective," IEEE Signal Processing Letters, 2015, vol. 22, pp. 938-942. http://dx.doi.org/10.1109/LSP.2014.2378264

[7] G. Zhang, Y. Xu, X. Wang and M. Guizani, "Multicast Capacity for Hybrid VANETS with Directional Antenna and Delay Constraint," IEEE Journal on Selected Area in Communications, 2012, vol. 30, pp. 818-833. http://dx.doi.org/10.1109/JSAC. 2012.120515

[8] T. Huang, Y. Teng, M. Liu and J. Liu, "Capacity Analysis for Cognitive Heterogeneous Network with Idea/Non-Idea Sensing," Frontiers of Information Technology \& Electronic Engineering, 2015, vol. 16, pp. 1-11. http://dx.doi.org/10.1631/FITEE.1400129

[9] B. Liu, Z. Liu and D. Towsley, "On the Capacity of Hybrid Wireless Networks," Proc. IEEE INFOCOM, San Francisco, Canada, 2003, pp. 1543-1552. http://dx.doi.org/10.1109/infcom. 2003.1208989

[10] U. C. Kozat and L. Tassiulas, "Throughput Capacity of Random Ad Hoc Networks with Infrastructure Support" Proc. ACM MobiCom, San Diego, Canada, 2003, pp. 55-65. http://dx.doi.org/10.1145/938985.938992

[11] A. Zemliavov and G. Veciana, "Capacity of Ad Hoc Wireless Networks with Infrastructure Support," IEEE Journal on Selected Areas in Communications, 2005, vol. 23, pp. 657-667. http://dx.doi.org/10.1109/JSAC.2004.842536

[12] E. Perevalov, R. S. Blum and D. Safi, "Capacity of Clustered Ad Hoc Networks: How Large is Large?" IEEE Transactions on Communication, 2006, vol. 54, pp. 1672-1681. http://dx.doi.org/10.1109/TCOMM.2006.878836

[13] G. Alfano, M. Garetto and E. Eleonardi, "Capacity Scaling of Wireless Networks with Inhomogeneous Node Density: Upper Bounds," IEEE Journal on Selected Areas in Communications, 2009, vol. 27, pp. 1147-1157. http://dx.doi.org/10.1109/JSAC. 2009.090911

[14] G. Alfano, M. Garetto and E. Eleonardi, "Capacity Scaling of Wireless Networks with Inhomogeneous Node Density: Lower Bounds," IEEE/ACM Transactions on Networking, 2010, vol. 18, pp. 1624-1636. http://dx.doi.org/10.1109/TNET.2010.2048719

[15] G. Gupta, and P. R. Kumar, "Internets in the Sky: Capacity of 3D Networks," Proc. IEEE Conference on Decision and Control, Sydney, Australia, 2000, pp. 2290-2295.

[16] L. Pan, P. Miao and F. Yuguan, "The Capacity Analysis of ThreeDimensional Wireless Ad Hoc Networks," IEEE/ACM Transactions on Networking, 2012, vol. 20, pp. 1304-1315. http://dx.doi.org/10.1109/TNET.2011.2178123

\section{AUTHORS}

Feng Wu. He is with School of Electronic Science and Engineering, National University of Defense Technology, Changsha, China (e-mail: 181911883@qq.com).

Jiang Zhu. He is a professor of National University of Defense Technology, Changsha, China

Yilong Tian. He is with School of Computer, National University of Defense Technology, Changsha, China

Zhipeng Xi. He is with School of Electronic Science and Engineering, National University of Defense Technology, Changsha, China.

This work was supported in part by National Natural Science Foundation of China (No.61401476 and No.61201166). Submittted 21 September 2015. Published as resubmitted by the authors 20 October 2015. 